\title{
Aldose reductase deficiency inhibits LPS-induced $M 1$ response in macrophages by activating autophagy
}

Peng Cheng ${ }^{1,2^{*}} \mathbb{0}$, Jianwei Xie ${ }^{1}$, Zhiyong Liu ${ }^{1}$ and Jian Wang ${ }^{2^{*}}$

\begin{abstract}
Macrophage M1 polarization mediates inflammatory responses and tissue damage. Recently, aldose reductase (AR) has been shown to play a critical role in M1 polarization in macrophages. However, the underlying mechanisms are unknown. Here, we demonstrated, for the first time, that AR deficiency repressed the induction of inducible nitric oxide synthase in lipopolysaccharide (LPS)-stimulated macrophages via activation of autophagy. This suppression was related to a defect in the inhibitor of nuclear factor KB (NF-KB) kinase (IKK) complex in the classical NF-KB pathway. However, the mRNA levels of IKK $\beta$ and IKKY were not reduced in LPS-treated AR knockout (KO) macrophages, indicating that their proteins were downregulated at the post-transcriptional level. We discovered that LPS stimuli induced the recruitment of more beclin1 and increased autophagosome formation in AR-deficient macrophages. Blocking autophagy through 3-methyladenine and ammonium chloride treatment restored IKK $\beta$ and IKKY protein levels and increased nitric oxide synthase production in LPS-stimulated AR-deficient macrophages. More assembled IKK $\beta$ and IKKY underwent ubiquitination and recruited the autophagic adaptor p62 in LPS-induced AR KO macrophages, promoting their delivery to autophagosomes and lysosomes. Collectively, these findings suggest that AR deficiency is involved in the regulation of NF-KB signaling, and extends the role of selective autophagy in fine-tuned M1 macrophage polarization.
\end{abstract}

Keywords: Aldose reductase, Bone marrow-derived macrophages, Macrophage polarization, Autophagy, Ubiquitination, Nuclear factor $\mathrm{kB}$

\section{Introduction}

Macrophages demonstrate significant plasticity and can modify their phenotype and function in response to their microenvironment [1]. Macrophages are roughly categorized into two different subsets, namely, inflammatory M1 and anti-inflammatory M2 macrophages [2]. In response to lipopolysaccharide (LPS) treatment, either alone or in combination with pro-inflammatory cytokines such as interferon- $\gamma$, macrophages undergo

\footnotetext{
*Correspondence: chengpengfmmu@126.com; jwangfm@fmmu.edu.cn ${ }^{1}$ Department of Neurology, Second Naval Hospital of Southern Theater Command (425th Hospital of the People's Liberation Army), Sanya, 572000, China ${ }^{2}$ Institute of Neurosciences, Fourth Military Medical University, Xi'an 710032, China
}

M1 polarization, characterized by the expression of proinflammatory cytokines and cytotoxic mediators (reactive oxygen and nitrogen species), as well as increased phagocytic and antigen-presenting capacity [3].

Aldose reductase (AR), a rate-limiting enzyme in the polyol pathway that catalyzes the reduction of glucose to sorbitol in the presence of reduced nicotinamide adenine dinucleotide phosphate, has emerged as a molecular target in multiple inflammatory diseases [4]. Ravindranath et al. demonstrated that transgenic mice overexpressing AR showed a more pronounced inflammatory response in a cecal ligation and puncture model [5]. In addition, AR inhibition suppresses inflammatory disorders or immune responses in several other animal 
models [6-10]. It was reported that M1-polarized human monocyte-derived macrophages expressed significantly higher levels of AR mRNA and AR protein compared with M2-polarized macrophages in vitro [11]. Accumulating evidence implicates the classical nuclear factor- $\mathrm{kB}$ (NF- $\mathrm{kB}$ ) signaling pathway in the modulation of M1 macrophage polarization [12-15]. Previously, Zhang et al. reported that the expression of AR is upregulated after spinal cord injury in wild type (WT) mice while phosphorylated NF- $\mathrm{kB}$ is downregulated, and that the number of M1-like macrophages is decreased after spinal cord injury in AR knockout (KO) mice [16]. However, the exact mechanisms involved are unknown.

Recently, it has been reported that AR deficiency or inhibition enhances autophagy in mouse cardiac myocytes under pathological cardiac hypertrophy or fasting conditions $[17,18]$. Autophagy is an essential cell-intrinsic mechanism that affords protection against starvation. Moreover, it represents a quality control system that can deliver damaged organelles, worn-out or misfolded proteins, and invading microorganisms from the cytoplasm to the lysosomes for degradation [19]. Defects in autophagy are linked to many human diseases and to the function of cells of the immune response [20]. Experimentally, Toll-like receptor 4 (TLR4) is a sensor for autophagy associated with innate immunity in macrophages [21].

Herein, we demonstrate that LPS treatment leads to a defect at the IKK complex level and the production of more autophagosomes in AR-deficient macrophages. These findings prompted us to investigate the inherent relationships between these processes.

\section{Materials and methods}

\section{Isolation and culture of BMMs}

WT and AR KO C57BL/6 J mice were bred at the Laboratory Animal Center of Fourth Military Medical University. Bone marrow cells were isolated from adult mice and processed as described previously [22]. Then, the cells were cultured in DMEM (Gibco, Carlsbad, CA, USA) supplemented with $0.001 \% \beta$-mercaptoethanol, $1 \%$ penicillin/streptomycin, 1\% HEPES, 10\% FBS (Gibco), and $20 \%$ sL929 supernatant obtained from sL929 cells, which secrete macrophage colony-stimulating factor (M-CSF) required for the promotion of hematopoietic stem cell differentiation into macrophages. After 7 days of incubation with the conditioned medium, the floating cells were removed, and the viable cells attached to flat-bottomed 6or 24-well plastic culture plates or $75 \mathrm{~cm}^{2}$ culture flasks (Nunc, Roskilde, Denmark) were used to obtain BMMs. Next, the spent culture media were changed to complete medium without sL929 cell culture supernatant, then the cells were further cultured for 3 days to restore the BMMs to their resting state. Flow cytometry analysis of surface antigens showed that $98 \%$ of the cultured cells expressed F4/80, a specific marker of macrophages (Fig. 1a).

\section{Reagents}

LPS (E. coli 0111:B4), ammonium chloride $\left(\mathrm{NH}_{4} \mathrm{Cl}\right)$, and 3-methyladenine (3-MA) were purchased from SigmaAldrich (St. Louis, MO, USA). The reagents were used at the indicated concentrations.

\section{Flow cytometry}

BMMs were incubated in blocking solution (rat serum, 20 min at $4{ }^{\circ} \mathrm{C}$ ) and were subsequently stained with FITCconjugated anti-F4/80 (1:100, AbD Serotec, Hercules, CA, USA) in the dark for $20 \mathrm{~min}$ at $4{ }^{\circ} \mathrm{C}$. Acquisitions were performed on a Millipore flow cytometer (Guawa $6 \mathrm{HT})$. Subsequent data analyses were completed using FlowJo software version 7.6.2 (Tree Star, Ashland, OR, USA). Results are expressed as \% of positive cells.

\section{Immunofluorescence}

Cells were cultured to $~ 70-80 \%$ confluence using glass coverslips in 24-well plates. After treatment, primary BMMs were fixed with $4 \%$ paraformaldehyde in phosphate-buffered saline (PBS, $\mathrm{pH}=7.4$ ) for $30 \mathrm{~min}$ and then washed thrice with PBS. Non-specific antibody binding was blocked by incubating the cells for $1 \mathrm{~h}$ at room temperature $\left(20-25{ }^{\circ} \mathrm{C}\right)$ in PBS containing $5 \%$ bovine serum albumin and $0.3 \%$ Triton X-100. The cells were then stained with the following primary antibodies overnight at $4{ }^{\circ} \mathrm{C}$ : mouse anti-AR $(1: 200$, Santa Cruz Biotechnology, Santa Cruz, CA, USA), mouse anti-beclin1 (1:200, Santa Cruz Biotechnology), goat anti-microtubule-associated protein 1 light chain $3 \beta$ (LC3 $\beta, 1: 200$, Santa Cruz Biotechnology), mouse anti-lysosome-associated membrane protein 1 (LAMP1, 1:200, Santa Cruz Biotechnology), rabbit anti-IKK $\beta$ (1:100, Epitomics, Burlingame, CA, USA), rabbit anti-IKKY (1:200, Santa Cruz Biotechnology,), goat anti-p62 (1:200, Santa Cruz Biotechnology), and mouse anti-ubiquitin specific for K63 (PloyUb, 1:400, Abcam, Cambridge, UK). After washing with PBS, the cells were incubated for $1 \mathrm{~h}$ at room temperature in the dark with their respective secondary antibodies: fluorescein isothiocyanate-, DyLight 488-, and DyLight 594-conjugated secondary antibodies (1:500 800, Jackson ImmunoResearch Laboratories, West Grove, PA, USA). Diamidinophenylindole (DAPI, $1 \mu \mathrm{g} / \mathrm{ml}$, SigmaAldrich) was applied to visualize the nuclei. Rhodaminephalloidin (1:250, Life Technologies-Invitrogen, Carlsbad, USA) was used to stain the actin cytoskeleton of the cells. The cells were observed using a laser confocal microscope (FluoView FV1000 MPE, Olympus Corporation, 


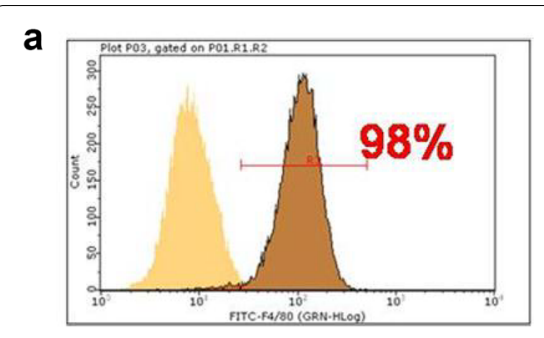

b
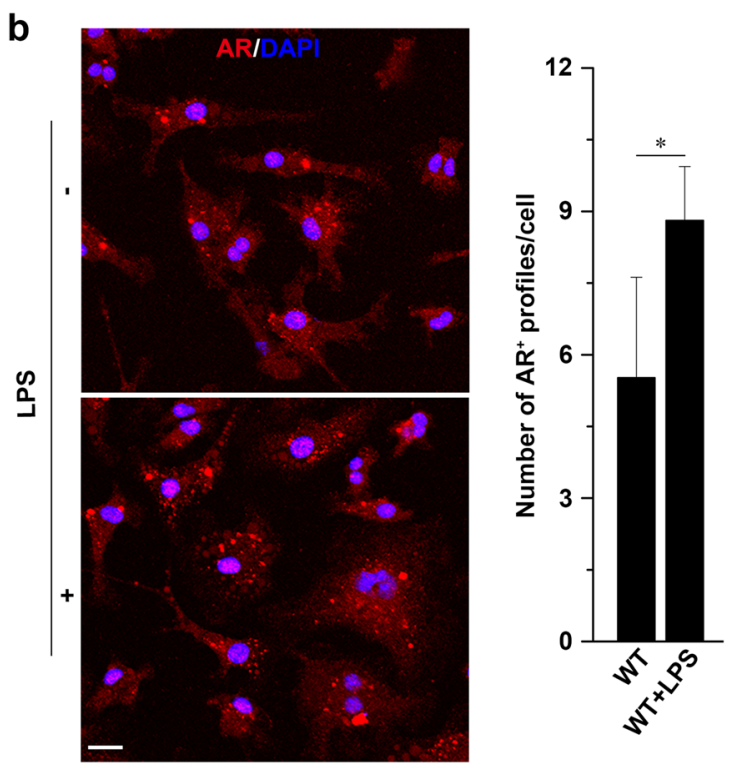

.

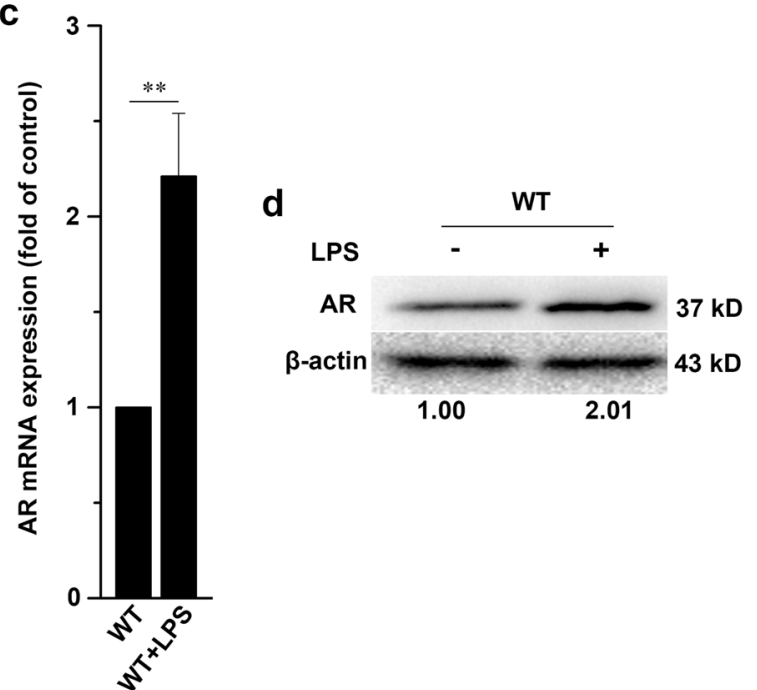

Fig. 1 Identification of BMMs in vitro and LPS stimulation upregulates AR expression in BMMs. a Following induction of BMMs from the bone marrow, approximately $98 \%$ of the cells were F4/80-positive. b Confocal microscopy of BMMs from WT mice left untreated or treated for $24 \mathrm{~h}$ with LPS (500 $\mathrm{ng} / \mathrm{ml})$, and then immunostained for AR. Scale bars $=10 \mu \mathrm{m}$. Right graph shows quantification analysis of the number of AR + foci per cell. $\mathbf{c}, \mathbf{d}$ mRNA and protein levels of AR in BMMs from WT mice left untreated or treated with $500 \mathrm{ng} / \mathrm{ml}$ LPS for $24 \mathrm{~h}$. Transcription levels are expressed as fold change of control and densitometric $A R / \beta$-actin ratios are shown below the blot. Data are expressed as the mean \pm SD from three independent experiments, ${ }^{*} P<0.05,{ }^{* *} P<0.01$
Tokyo, Japan). We applied the JACoP plugin of Image J (National Institutes Health, Bethesda, MD, USA) to perform co-localization analysis of any image pair, and the co-localization rate of the green and red signals was evaluated using Manders' overlap coefficient [23].

\section{Western blotting}

The extraction of cellular proteins and their detection via western blotting were performed as previously described [21, 24]. Western blotting was performed using the following primary antibodies: mouse anti-AR, mouse anti-beclin1, goat anti-LC3 $\beta$, mouse anti-LAMP1, and rabbit anti-IKK $\gamma$ antibodies (1:500, Santa Cruz Biotechnology); mouse anti-TLR4, rabbit anti-iNOS, rabbit anti-IKK $\alpha$, and rabbit anti-4-HNE (1:1000, Abcam); rabbit anti-IKK $\beta$ and rabbit anti-phospho-IKB $\alpha$ antibodies (1:1000, Epitomics); rabbit anti-phospho-IKK $\alpha / \beta$, rabbit anti-ІкB $\alpha$, rabbit anti-p65, and rabbit anti-phospho-p65 antibodies (1:1000, Cell Signaling Technology, Danvers, MA, USA); and mouse anti- $\beta$-actin antibodies (1:8000, Sigma-Aldrich). The blots were then incubated with their respective secondary antibodies: horseradish peroxidaseconjugated goat anti-rabbit IgG, goat anti-mouse IgG (both 1:8000, Abcam), and donkey anti-goat IgG (1:5000, Santa Cruz Biotechnology). $\beta$-actin was used as the loading control. The immunoreactive bands were scanned using the Bio-Rad ChemiDoc ${ }^{\mathrm{TM}} \mathrm{XRS}^{+}$imager with Image Lab $^{\text {TM }}$ Software (Bio-Rad Laboratories, CA, USA). Band intensity was quantified using Quantity One software (Bio-Rad Laboratories).

\section{Quantitative reverse transcription-polymerase chain reaction (qRT-PCR)}

Total RNA from BMMs was isolated using TRIzol Reagent (Invitrogen, Carlsbad, CA, USA) according to the manufacturer's instructions. PCR was performed on a Bio-Rad CFX $96^{\text {TM }}$ Real-Time system (Bio-Rad Laboratories) employing a SYBR Green qPCR core reagent kit (DRR081A, Takara Bio Inc., Otsu, Japan). qRT-PCR was performed using the following primers, specific for mouse AR: forward primer, 5'-ACGGCTATGGAA CAACTA- $3^{\prime}$ and reverse primer, $5^{\prime}$-TGTGGCAGTATT CAATCAG-3'; mouse IKK $\beta$ : forward primer, 5'-GGA GCCTGGGAAATGAAAGAA- $3^{\prime}$ and reverse primer, 5'-GCCAGAGCCCTACCTGATTG-3'; mouse IKKү: forward primer, $5^{\prime}$-AAGCACCCCTGGAAGAACC- $3^{\prime}$ and reverse primer, $5^{\prime}$-CCTGCTCTGAAGGCAGAT GTA-3'; mouse iNOS: forward primer, $5^{\prime}$-CCCTTCAAT GGTTGGTACATGG-3' ${ }^{\prime}$ and reverse primer, $5^{\prime}-\mathrm{ACA}$ TTGATCTCCGTGACAGCC-3'; mouse CD86: forward primer, $5^{\prime}$-TTGTGTGTGTTCTGGAAACGGAG- ${ }^{\prime}$ and reverse primer, $5^{\prime}$ - AACTTAGAGGCTGTGTTGCTGG$3^{\prime}$; and mouse $\beta$-actin: forward primer, $5^{\prime}$-CGTGCGTGA 
CATCAAAGAGAA- ${ }^{\prime}$ and reverse primer, $5^{\prime}$-GCTCGT TGCCAATAGTGATGA-3'. Gene expression was normalized using $\beta$-actin as an internal control, and fold changes were calculated.

\section{Transmission electron microscopy}

Cells were grown to $~ 80 \%$ confluence in $75 \mathrm{~cm}^{2}$ culture flasks. After stimulation, BMMs were harvested and centrifuged at $1500 \times g$ for $10 \mathrm{~min}$. The pellets were fixed in a mixture of $4 \%$ paraformaldehyde and $0.05 \%$ glutaraldehyde, post-fixed in $0.5 \%$ osmium tetroxide, dehydrated in a graded ethanol series and propylene oxide, and embedded in epoxy resin. Ultrathin sections were obtained using an ultramicrotome (EM UC6, Leica Microsystems, Baden-Württemberg, Germany), mounted on mesh grids (6-8 sections/grid), and counter-stained with uranyl acetate and lead citrate. The sections were viewed using a transmission electron microscope (JEM-1230, JEOL, Tokyo, Japan) equipped with a Gatan digital camera.

\section{Statistical analysis}

All data are expressed as the mean \pm SD. Statistically significant differences between the mean values were determined using two-tailed Student's t-test. Differences at $P<0.05$ were considered statistically significant. ${ }^{*} P<0.05$; ** $P<0.01$.

\section{Results}

\section{LPS stimulation upregulates AR expression in BMMs}

It has been reported that AR can regulate inflammatory signals and immune responses in several animal disease models [5-8]. Since inflammatory signals and immune responses are tightly correlated with macrophage polarization, we investigated changes in the expression of AR upon LPS stimulation in BMMs from WT C57BL/6 mice.

We used immunofluorescence staining to label AR in BMMs. The number of AR-immunoreactive profiles in BMMs was significantly increased after treatment with LPS for $24 \mathrm{~h}$ (Fig. 1b). We next used qRT-PCR and western blotting to detect the changes of AR mRNA and protein in LPS-stimulated BMMs. The transcription and expression of AR were both significantly increased following treatment with LPS for $24 \mathrm{~h}$ (Fig. 1c, d). Collectively, these results indicate that AR expression is triggered upon stimulation of BMMs with LPS.

\section{AR deficiency suppresses the $M 1$ response and NF-KB} activation at the IKK complex level in LPS-stimulated BMMs In response to LPS treatment, macrophages undergo M1 polarization, characterized by the expression of inducible nitric oxide synthase (iNOS) [25]. Activation of the canonical NF- $\mathrm{KB}$ signaling pathway in response to LPS was demonstrated by monitoring iNOS expression, immunoblot analysis, and the measurement of nitric oxide (NO) production-important downstream products of LPS signaling in macrophages [26].

We hypothesized the existence of a relationship between AR and the NF- $\mathrm{kB}$ p 65 signaling pathway. To test this hypothesis, we carried out experiments on BMMs from WT or AR KO mice. Stimulation of BMMs with $500 \mathrm{ng} / \mathrm{ml}$ LPS increased the levels of iNOS in a time-dependent manner during the first $32 \mathrm{~h}$ (Fig. 2a). Expression of iNOS peaked at $16 \mathrm{~h}$ after LPS treatment (Fig. 2a). The induction of iNOS upon LPS stimulation decreased markedly when the AR gene was knocked out (Fig. 2a). These results suggested that the LPS-stimulated induction of iNOS was closely associated with AR.

For most inducers of the classical NF- $\mathrm{kB}$ signaling pathway, IKK $\alpha$ is not required for the phosphorylation of $\mathrm{I} \mathrm{B} \alpha$; however, genetic studies have shown that NF- $\mathrm{KB}$ p65 signaling requires IKK $\beta$ and IKK $\gamma$ to phosphoryl-

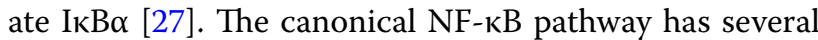
important components, including TLR4, IKK $\beta$, IKK $\gamma$, I $\mathrm{B} \alpha$, and $\mathrm{p} 65$ [28-30]. Following $16 \mathrm{~h}$ of LPS exposure, we detected lower levels of phospho-IKK $\alpha / \beta(\mathrm{p}-\mathrm{IKK} \alpha / \beta)$, IKK $\beta$, IKK $\gamma$, phospho-ІкB $\alpha$ (p-ІкB $\alpha)$, phospho-p65 (p-p65), and iNOS proteins in AR KO BMMs than in control cells (Fig. 2b). We used qRT-PCR to measure the mRNA levels of IKK $\beta$, IKK $\gamma$, iNOS, and CD68 in the total RNA obtained from the BMMs after $16 \mathrm{~h}$ of treatment with or without LPS. The results showed that mRNA levels of IKK $\beta$ and IKK $\gamma$ were not affected in the LPStreated AR KO BMMs (Fig. 2c). In AR KO macrophages, the mRNA levels of iNOS were less increased compared to that in WT cells after LPS stimulation (Fig. 2c). The mRNA level of CD86, another M1 marker, was also less increased in AR KO BMMS compared to that in WT cells after LPS treatment (Fig. 2c). The above data indicated that the suppression of the M1 response in AR-deficient macrophages after LPS stimulation was due to the posttranscriptional degradation of IKK $\beta$ and IKK $\gamma$. To determine the temporal patterns of change in the protein levels of IKK $\beta$ and IKK $\gamma$, we stimulated BMMs from WT or AR KO mice with LPS for $32 \mathrm{~h}$ and immunoblotted the lysates for IKK $\beta$ and IKK $\gamma$. The time-course experiments revealed that LPS induced a gradual reduction of IKK $\beta$ and IKK $\gamma$ proteins in BMMs from AR KO mice (Fig. 2d).

\section{AR deficiency enhances autophagosome formation and maturation in LPS-stimulated BMMs}

To determine whether autophagosome formation occurred because of AR deficiency, we treated BMMs from WT or AR KO mice with or without LPS. Beclin1 is a key component of the class III phosphatidylinositol 3-kinase (PI3K) complex, which initiates autophagosome formation [31]. Incubation of BMMs with $500 \mathrm{ng} / \mathrm{ml}$ LPS 


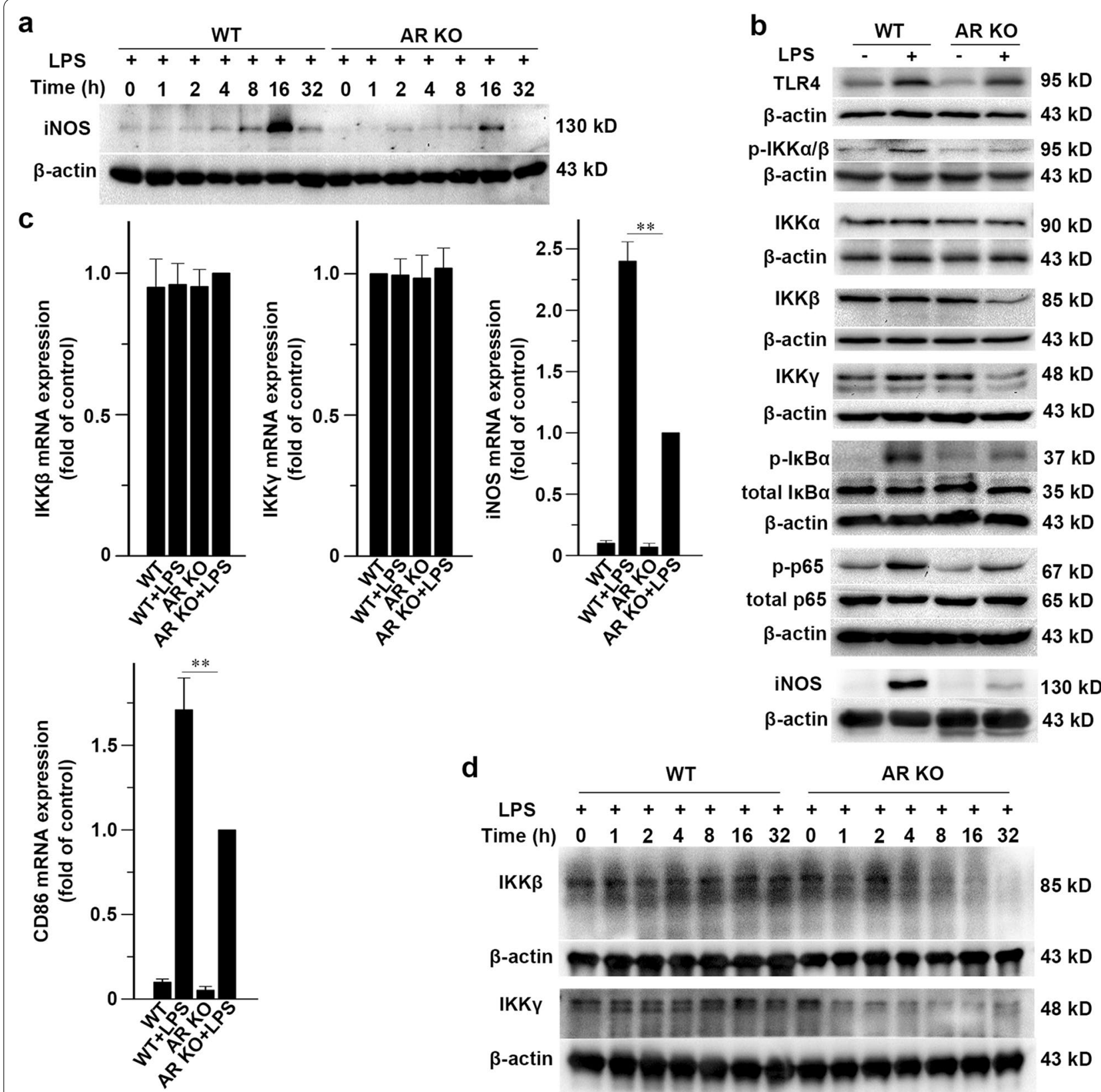

Fig. 2 Stimulation of AR-deficient macrophages with LPS decreases the protein levels of IKK $\beta$ and IKKY. a BMMs from WT or AR KO mice were treated with $500 \mathrm{ng} / \mathrm{ml}$ LPS for the indicated time periods, and the production of iNOS was assayed. $\mathbf{b}$ Effect of AR deficiency on several important components of the TLR4/NF-KB signaling pathway. Immunoblot analysis for TLR4, p-IKKa/ $\beta$, IKKa, IKKß, IKKY, IKBa, p-|KBa, p65, p-p65, and iNOS in BMMs from WT or AR KO mice treated with or without $500 \mathrm{ng} / \mathrm{ml}$ LPS for $16 \mathrm{~h}$. c qRT-PCR of IKKR, IKKY, iNOS, and CD86 mRNA in BMMs from WT or AR KO mice treated with or without $500 \mathrm{ng} / \mathrm{ml}$ LPS for $16 \mathrm{~h}$. Transcription levels are expressed as fold change of control. d BMMs from WT or AR KO mice were treated with $500 \mathrm{ng} / \mathrm{ml}$ LPS for the indicated time periods. Data represent the mean \pm SD of three different experiments

for $16 \mathrm{~h}$ led to the recruitment of beclin1, as shown in the change in beclin1 staining from a diffuse to a punctate pattern (Fig. 3a). We then conducted a detailed quantitative analysis of LPS-stimulated beclin1 aggregation. LPS induced a significant increase in the number of beclin $1^{+}$profiles in AR KO cells (Fig. 3a). The increase in beclin1 levels was also confirmed through immunoblotting (Fig. 3b).

In macroautophagy, cells form double-membraned vesicles, known as autophagosomes, around a portion of the cytoplasm [32]. Two forms of LC3, LC3I, and LC3II are produced post-transcriptionally; LC3I 


\section{(See figure on next page.)}

Fig. 3 AR deficiency enhances autophagosome formation and maturation in LPS-stimulated macrophages. a BMMs from WT or AR KO mice were treated with or without $500 \mathrm{ng} / \mathrm{ml}$ LPS for $16 \mathrm{~h}$ and then immunostained for beclin 1. Right graph represents quantification analysis of the number of beclin $1+$ foci per cell. Scale bars $=50 \mu \mathrm{m}$. $\mathbf{b}$ Immunoblot analysis of beclin 1 in BMMs from WT or AR KO mice treated with or without $500 \mathrm{ng} / \mathrm{ml}$ LPS for $16 \mathrm{~h}$. c Induction of LC3 + vacuoles in BMMs from WT or AR KO mice treated with or without $500 \mathrm{ng} / \mathrm{ml}$ LPS for $16 \mathrm{~h}$ and then immunostained for LC3. Right graph represents the quantification analysis of the number of LC3 + puncta per cell. Scale bars $=20 \mu \mathrm{m}$. $\mathbf{d}$ Immunoblot analysis of LC3 in BMMs from WT or AR KO mice treated with or without $500 \mathrm{ng} / \mathrm{ml} \mathrm{LPS} \mathrm{for} 16 \mathrm{~h}$. Densitometric LC3II/LC3I/ $\beta$-actin ratios are shown below the blot. e Time-course analysis of autophagy in macrophages during the $32 \mathrm{~h}$ of LPS stimulation. BMMs from WT or AR KO mice were treated with $500 \mathrm{ng} / \mathrm{ml}$ LPS for the indicated durations. Densitometric LC3II/LC3I/ $\beta$-actin ratios are shown below the blot. $\mathbf{f} / \mathrm{mm} u n o b l o t$ analysis of LAMP1 in BMMs from WT or AR KO mice treated with or without LPS for $16 \mathrm{~h}$. $\mathbf{g}$ Confocal microscopy of BMMs from WT or AR KO mice treated with or without $500 \mathrm{ng} / \mathrm{ml}$ LPS for $16 \mathrm{~h}$ and immunostained for LC3 and LAMP1. Scale bars $=20 \mu \mathrm{m}$. Right graphs represent co-localization of LAMP1 and LC3 signals evaluated using Manders' overlap coefficient. $\mathbf{h}$ Ultrastructural analysis of autophagy via transmission electron microscopy in BMMs. WT or AR KO BMMs were treated with or without $500 \mathrm{ng} / \mathrm{ml} \mathrm{LPS} \mathrm{for} 16 \mathrm{~h}$. The panel on the right is the enlargement of the outlined area on the left. Scale bars $=1 \mu \mathrm{m}$. Right graphs represent the quantification of the number of autophagosomes and autolysosomes per cross-sectioned cell. Data are expressed as the mean \pm SD from three independent experiments, ${ }^{*} P<0.05,{ }^{*} P<0.01$

is cytosolic, whereas LC3II is membrane-bound and is mainly enriched in the autophagosomal vacuoles, and the amount of LC3II is correlated with the extent of the autophagosome formation [33, 34]. The autophagic vacuoles can be imaged using confocal microscopy via immunofluorescence staining for LC3, which delineates them in the cytoplasm [35]. We then conducted a detailed quantitative analysis of LPS-stimulated autophagy. Without LPS treatment, AR KO macrophages exhibited more $\mathrm{LC}^{+}$vacuoles compared to WT cells (Fig. 3c). After LPS induction, there was a significant increase in the number of autophagosomes in AR KO BMMs (Fig. 3c). The increase in autophagic flux was confirmed by immunoblotting with an LC3 antibody (Fig. 3d). To observe the temporal pattern of autophagy within $32 \mathrm{~h}$, we immunoblotted lysates from LPS-stimulated WT or AR KO BMMs for LC3. The time-course experiment showed that LPS treatment significantly increased autophagic flux in AR KO cells compared with WT controls within $32 \mathrm{~h}$ (Fig. 3e).

As their maturation proceeds, autophagosomes fuse with lysosomes, resulting in the degradation of their contents [36]. LAMP1 is widely used as a lysosome marker. The merging of LC3 with LAMP1 indicates the presence of mature autophagosomes (autolysosomes). Immunoblotting showed that LAMP1 was significantly upregulated in LPS-stimulated AR KO cells (Fig. 3f). In AR KO BMMs, LPS promoted the maturation of autophagosomes as evidenced by an increase in the colocalization of LC3 with LAMP1 (Fig. 3g).

Transmission electron microscopy was also used to examine the effects of LPS on autophagy in BMMs from WT or AR KO mice. The number of double-membrane vacuoles, a typical feature of autophagosomes, was markedly increased in non-stimulated AR KO macrophages compared with that in WT cells; however, the fusion of primary lysosomes and autophagosomes, a typical feature of autolysosomes, was rare in AR KO and WT macrophages without LPS stimulation (Fig. 3h). Furthermore, it was observed that LPS treatment induced more autophagosomes and autolysosomes in AR-deficient cells compared to the controls (Fig. 3h).

Collectively, these results indicate that AR deficiency promotes autophagosome biogenesis in non-stimulated 


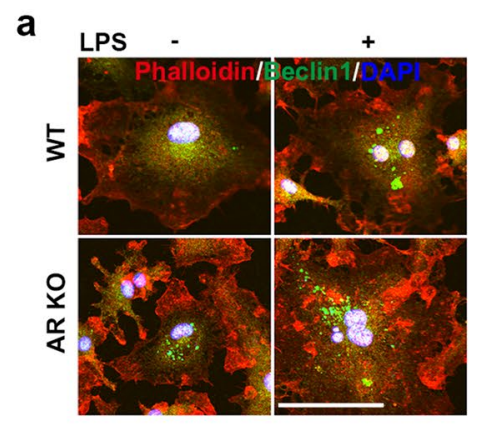

b
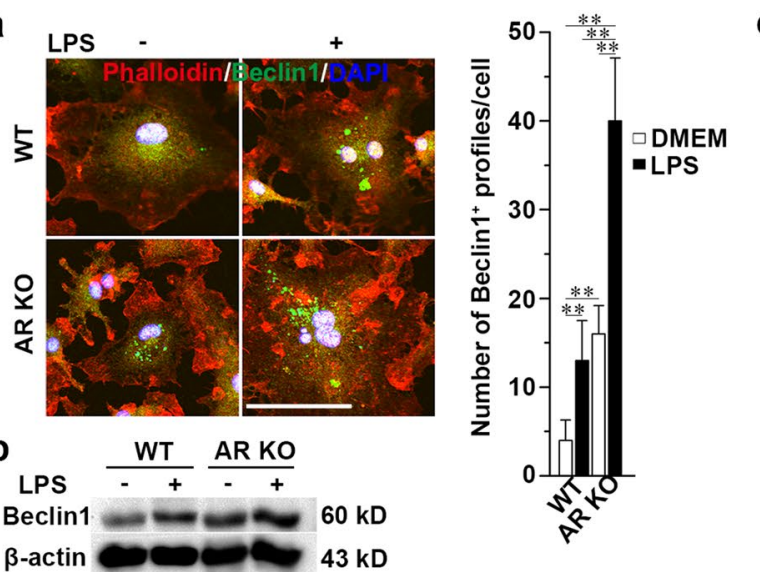
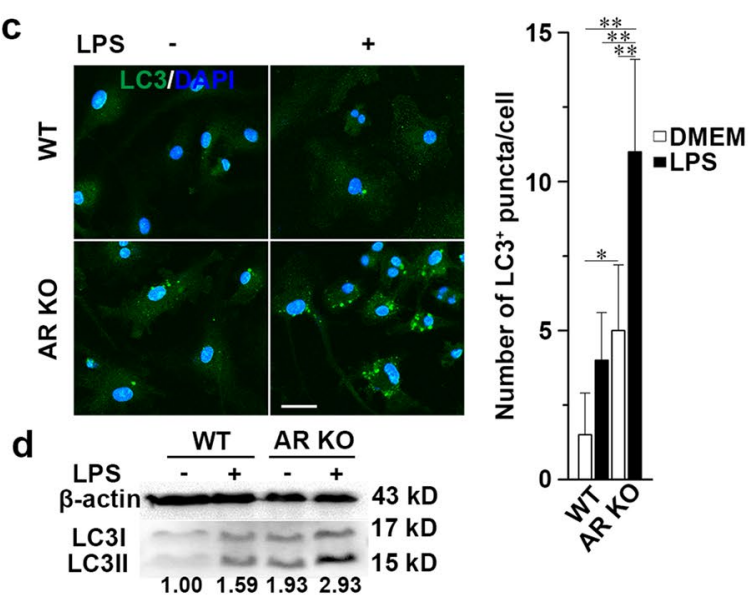

e
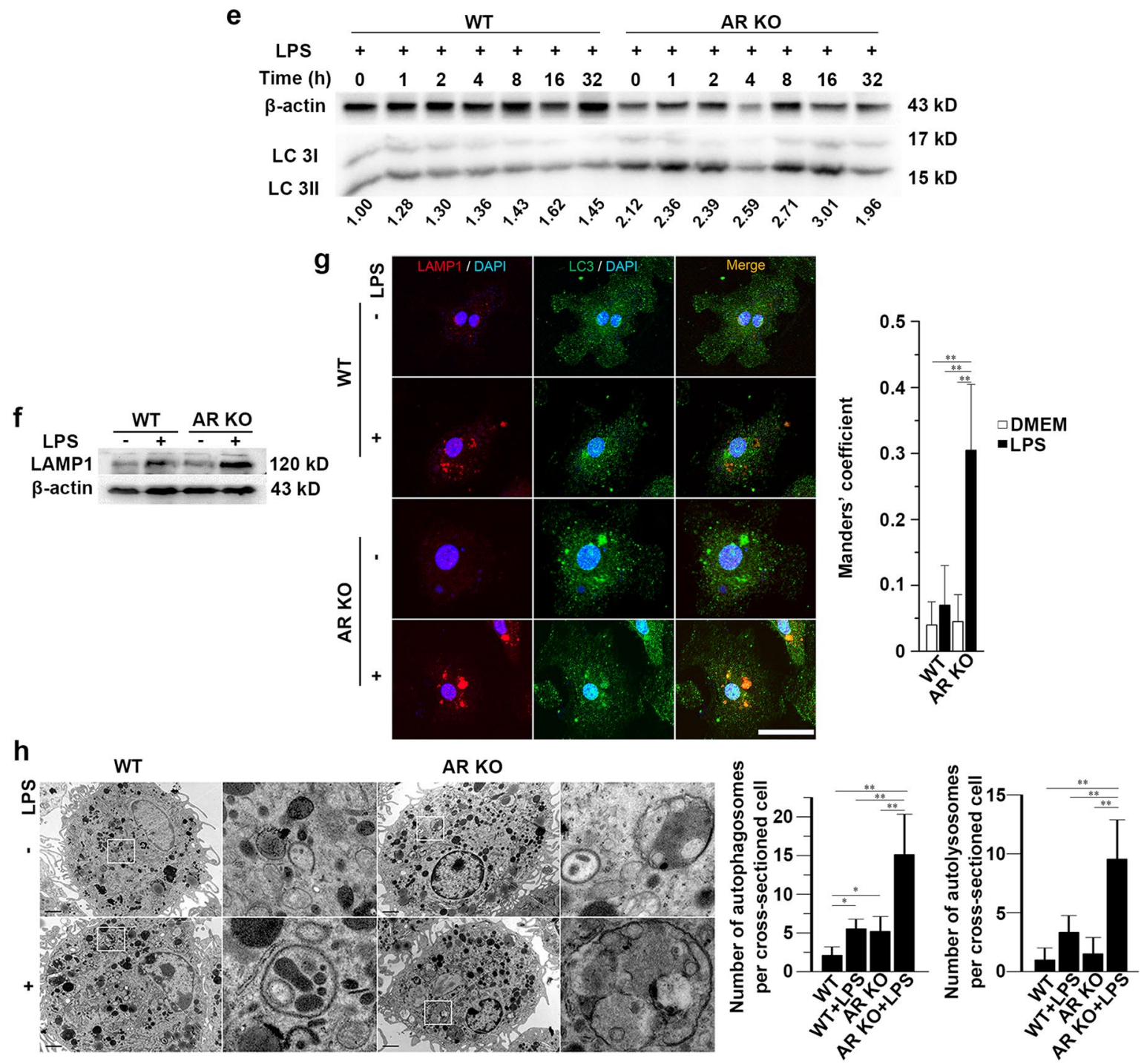

Fig. 3 (See legend on previous page.) 


\section{(See figure on next page.)}

Fig. 4 AR deficiency mediates the autophagic degradation of IKK $\beta$ and IKKY in LPS-stimulated macrophages. a, c WT or AR KO BMMs were incubated for $16 \mathrm{~h}$ in the presence of LPS $(500 \mathrm{ng} / \mathrm{ml})$, LPS +3-MA $(3 \mathrm{mM})$, or LPS + NH ${ }_{4} \mathrm{Cl}(10 \mathrm{mM})$, followed by immunostaining for LC3. Lower graphs represent the quantification of the number of autophagosomes per cell. Scale bars $=20 \mu \mathrm{m}$. b, d Immunoblot analysis for $L C 3$, IKK $\beta$, IKKY, and iNOS in lysates of WT or AR KO BMMs treated with $500 \mathrm{ng} / \mathrm{ml}$ LPS for $16 \mathrm{~h}$ in the presence or absence of 3-MA (3 mM) or NH${ }_{4} \mathrm{Cl}(10 \mathrm{mM})$. Densitometric LC3II/LC3I/ $\beta$-actin ratios are shown below the blot. Data are expressed as the mean $\pm S D$ from three independent experiments. ${ }^{*} P<0.05,{ }^{* *} P<0.01$

macrophages, and further strengthens biogenesis and maturation in LPS-activated BMMs.

\section{AR deficiency mediates autophagic degradation of IKK $\beta$ and IKK $\gamma$ in LPS-treated BMMs}

The addition of the PI3K inhibitor 3-MA has been shown to block autophagosome formation [37]. Our results showed that 3-MA (3 mM) was able to block LPS-stimulated autophagy as detected by LC3 immunofluorescence (Fig. 4a) and immunoblotting (Fig. 4 b). $\mathrm{NH}_{4} \mathrm{Cl}$ is a commonly used lysosome inhibitor. It localizes to acidic vesicles and impairs lysosomal acidification and protease activity, resulting in the accumulation of autophagosomes by impairing their fusion with lysosomes [38]. Incubation of the cells with $10 \mathrm{mM} \mathrm{NH}_{4} \mathrm{Cl}$ for $16 \mathrm{~h}$ resulted in the accumulation of $\mathrm{LC}^{+}$vacuoles (Fig. 4c) and a large increase in LC3II levels (Fig. 4d) in LPS-stimulated macrophages.

To investigate the mechanisms underlying the observed downregulation of key components of the IKK complex and enhanced autophagy, we analyzed the protein levels of IKK $\beta$ and IKK $\gamma$ via immunoblotting in LPS-stimulated BMMs after inhibiting autophagy using 3-MA. Our results revealed that the protein levels of IKK $\beta$ and $\mathrm{IKK} \gamma$ were rescued following 3-MA administration in LPS-treated AR-deficient cells (Fig. 4c). The induction of iNOS in LPS-treated AR KO macrophages was also significantly increased after 3-MA treatment (Fig. 4c). This was further supported by our data showing that the protein levels of IKK $\beta$ and IKK $\gamma$ were restored as reflected by the increased levels of iNOS in LPS-stimulated AR-deficient cells upon addition of $\mathrm{NH}_{4} \mathrm{Cl}$ (Fig. 4d). These results suggest that IKK $\beta$ and IKK $\gamma$ were loaded into autophagosomes and subsequently degraded in lysosomes, which limited iNOS induction and M1 polarization in LPS-activated AR-deficient BMMs.
AR deficiency facilitates IKK $\beta$ and IKK $\gamma$ to co-localize with autophagosomes and lysosomes in LPS-stimulated BMMs

In the initial phase of autophagy, beclin1 serves as a platform to recruit autophagy activators and inhibitors [39]. We immunostained BMMs for IKK $\beta$, IKK $\gamma$, and beclin1 after $1 \mathrm{~h}$ of incubation with LPS. We showed that more intracellular structures were labeled with IKK $\beta$ and IKK $\gamma$ co-localized with beclin $1^{+}$foci in AR KO BMMs after exposure to LPS (Fig. 5a, b). These results indicate that in the initial induction of autophagy, IKK $\beta$ and IKK $\gamma$ are recruited by beclin1.

To verify that IKK $\beta$ entered the autophagosomes, we performed immunocytochemistry to label IKK $\beta$ and autophagic vacuoles. We observed autophagosome formation $1 \mathrm{~h}$ after treatment with LPS. Autophagosome induction was poor and only a few overlapping IKK $\beta$ labeled structures and LC3-labeled puncta were observed in resting macrophages (Fig. 5c). Upon LPS stimulation, a higher number of IKK $\beta^{+}$structures co-localized with $\mathrm{LC}^{+}$vacuoles was observed in AR KO macrophages than in WT macrophages (Fig. 5c). Similarly, AR knockout increased LC3 staining and its co-localization with IKK $\gamma$ in LPS-treated BMMs (Fig. 5d). Next, we detected higher levels of co-localization between the lysosome marker LAMP1 and IKK $\beta$ and IKK $\gamma$ in LPS-induced AR KO BMMs (Fig. 5e, f). These results indicate that after recruitment by beclin1, IKK $\beta$ and IKK $\gamma$ can be engulfed by autophagosomes and probably be degraded after fusion with lysosomes.

\section{AR deficiency, polyubiquitination, and p62 co-mediate the autophagic degradation of IKK $\beta$ and IKKY in LPS-stimulated BMMS}

Autophagic adaptors represent a mechanism through which intracellular targets are delivered to 


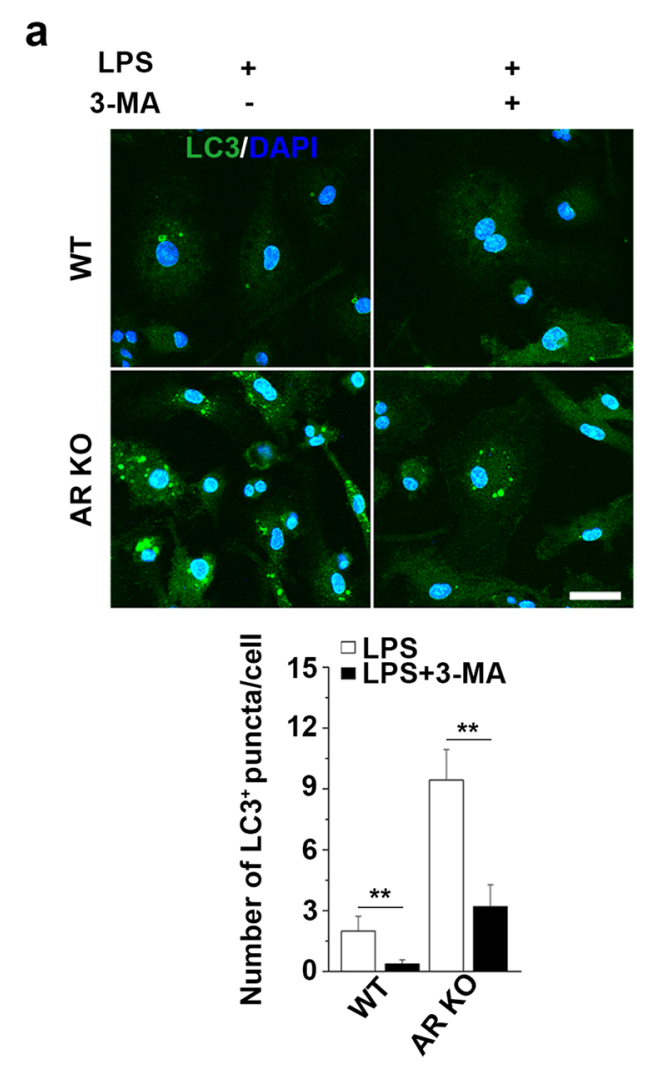

\section{C}
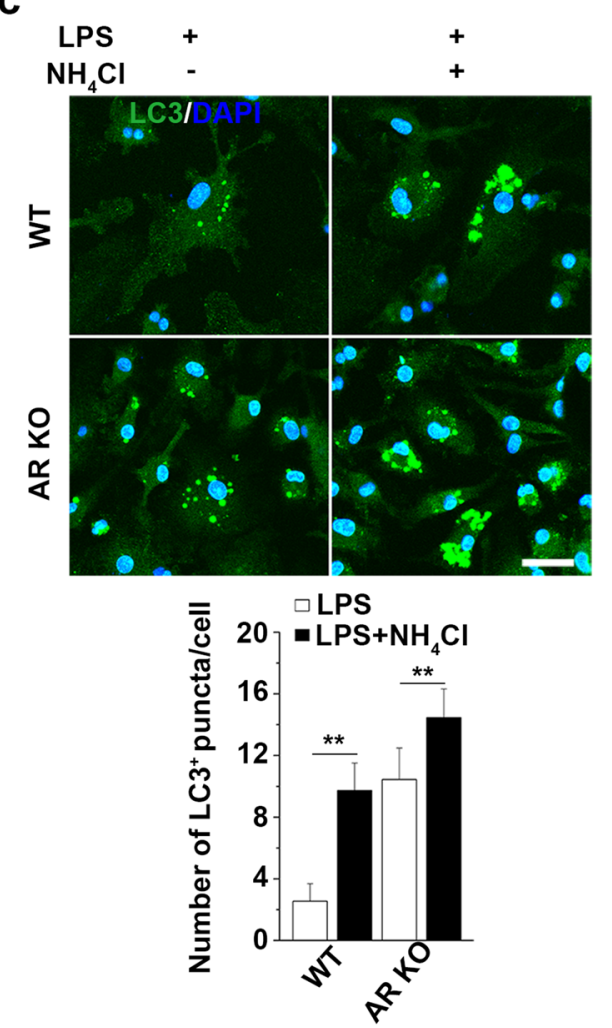

b
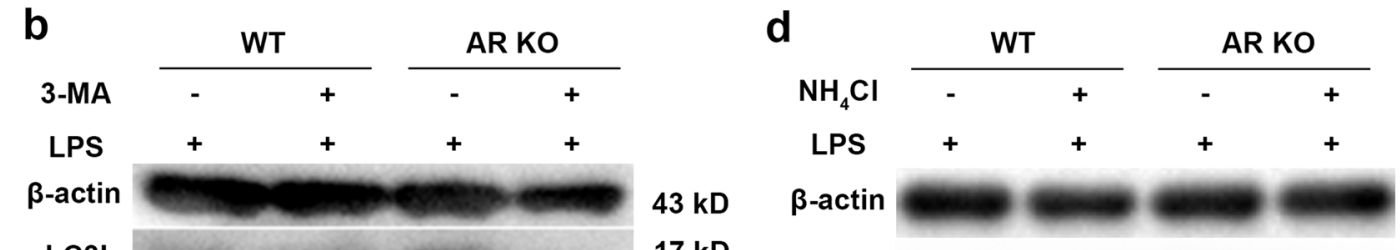

$43 \mathrm{kD}$
$17 \mathrm{kD}$

$\beta$-actin

$43 \mathrm{kD}$

LC3I



$17 \mathrm{kD}$

LC3II

$15 \mathrm{kD}$

$15 \mathrm{kD}$

(n)

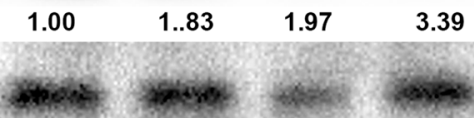

$85 \mathrm{kD}$

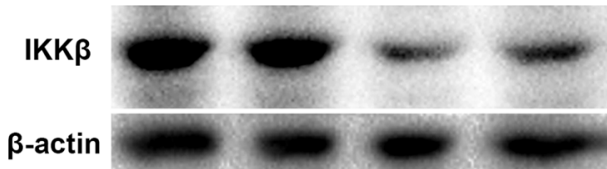

$85 \mathrm{kD}$

IKK $\beta$

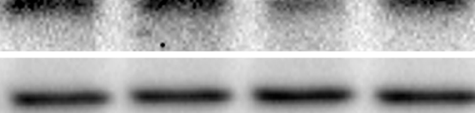

$43 \mathrm{kD}$

$43 \mathrm{kD} \quad \beta$-actin

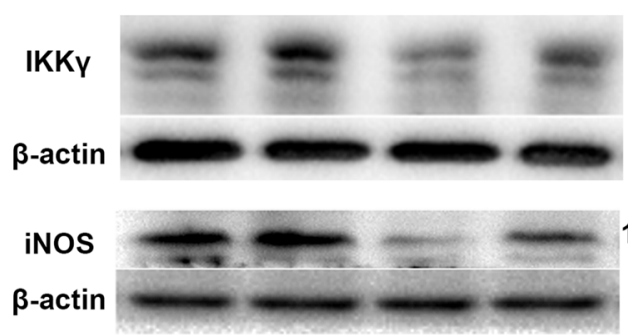

48 kD IKKY

$\beta$-actin

$48 \mathrm{kD}$

$43 \mathrm{kD}$

$130 \mathrm{kD} \quad \mathrm{iNOS}$

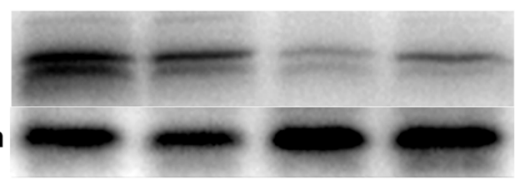

$43 \mathrm{kD}$

$43 \mathrm{kD}$

$\beta$-actin

$43 k D$ 

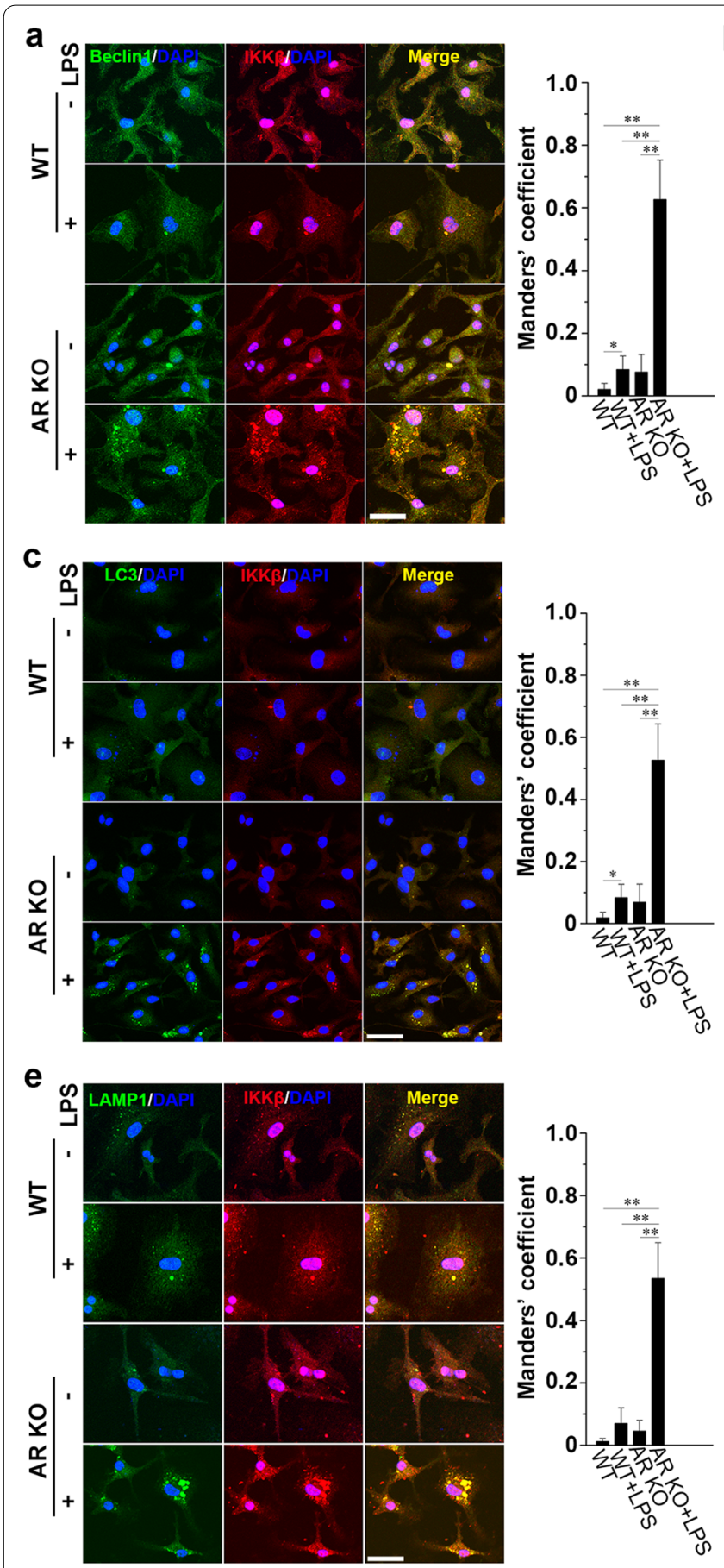
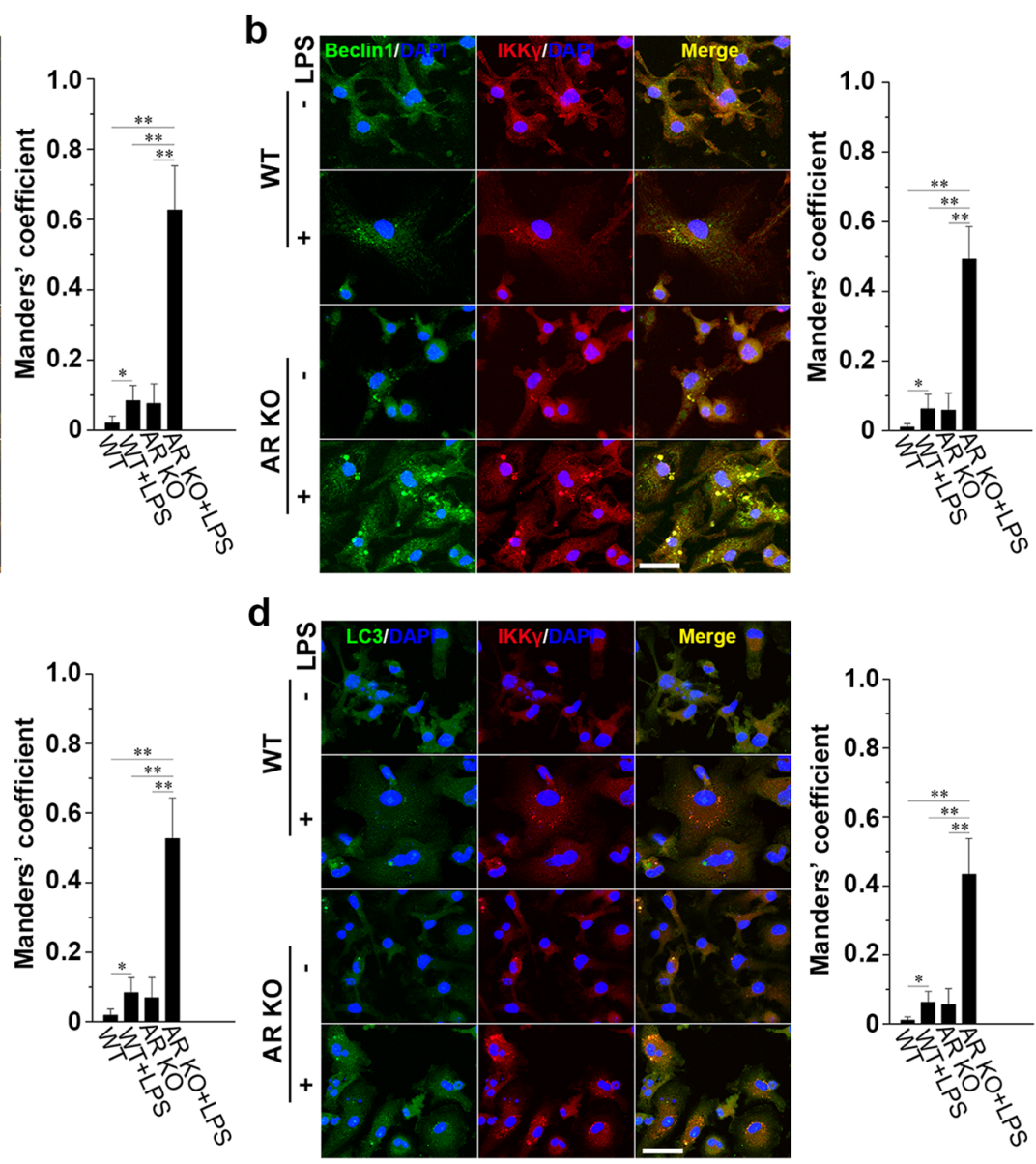

\section{f}
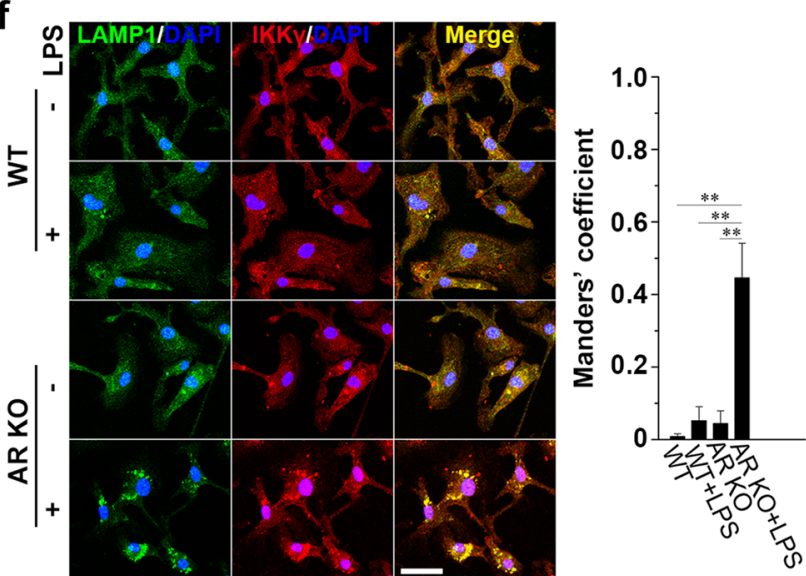

Fig. 5 AR deficiency facilitates IKK $\beta$ and IKKY to co-localize with autophagosomes and lysosomes in LPS-stimulated macrophages. Confocal microscopy of BMMs from WT or AR KO mice treated with or without $500 \mathrm{ng} / \mathrm{ml}$ LPS $1 \mathrm{~h}$ and co-stained for $\mathbf{a}$ beclin 1 and IKK $\beta$, $\mathbf{b}$ beclin 1 and $I K K Y, \mathbf{c} L C 3$ and IKKB, $\mathbf{d} L C 3$ and IKKY, e LAMP1 and IKKß, and $\mathbf{f} L A M P 1$ and IKKY. Scale bars $=20 \mu \mathrm{m}$. Right graphs represent the quantification of co-localization of signals using Manders' overlap coefficient. Data are expressed as the mean $\pm \mathrm{SD}$ from three independent experiments, ${ }^{*} P<0.05$, ${ }^{*} P<0.01$

autophagosomes [40]. An adaptor protein, p62, recognizes polyubiquitinated targets and binds to the ubiquitin-like autophagosome membrane LC3 in the autophagic degradation pathway [41]. It has been shown that TLR4-mediated autophagy is a p62-dependent type of selective autophagy in macrophages [42]. K63-linked 

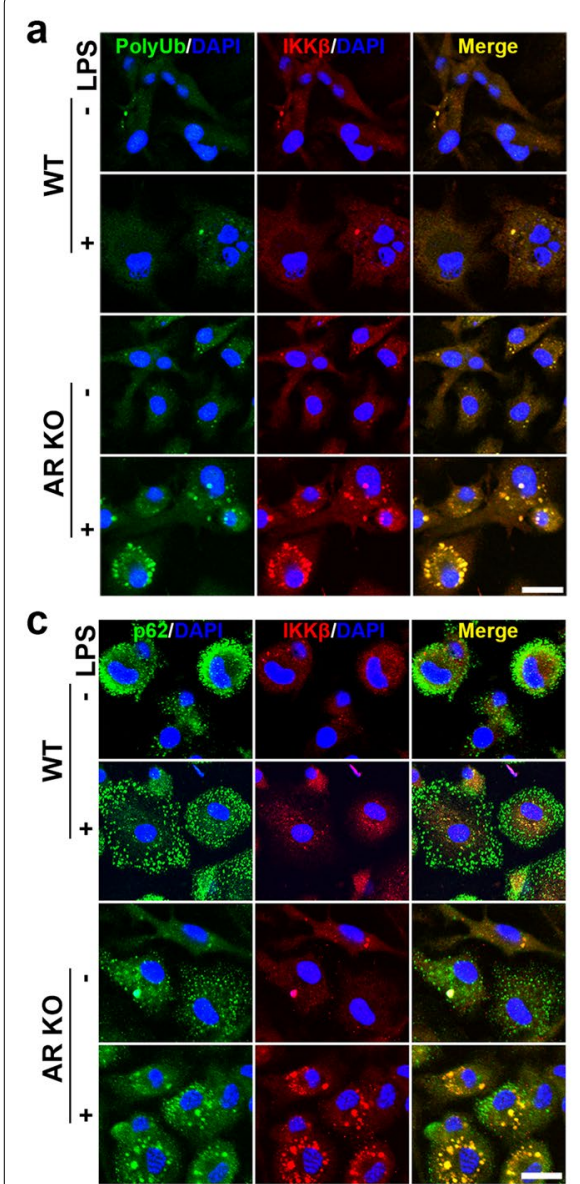
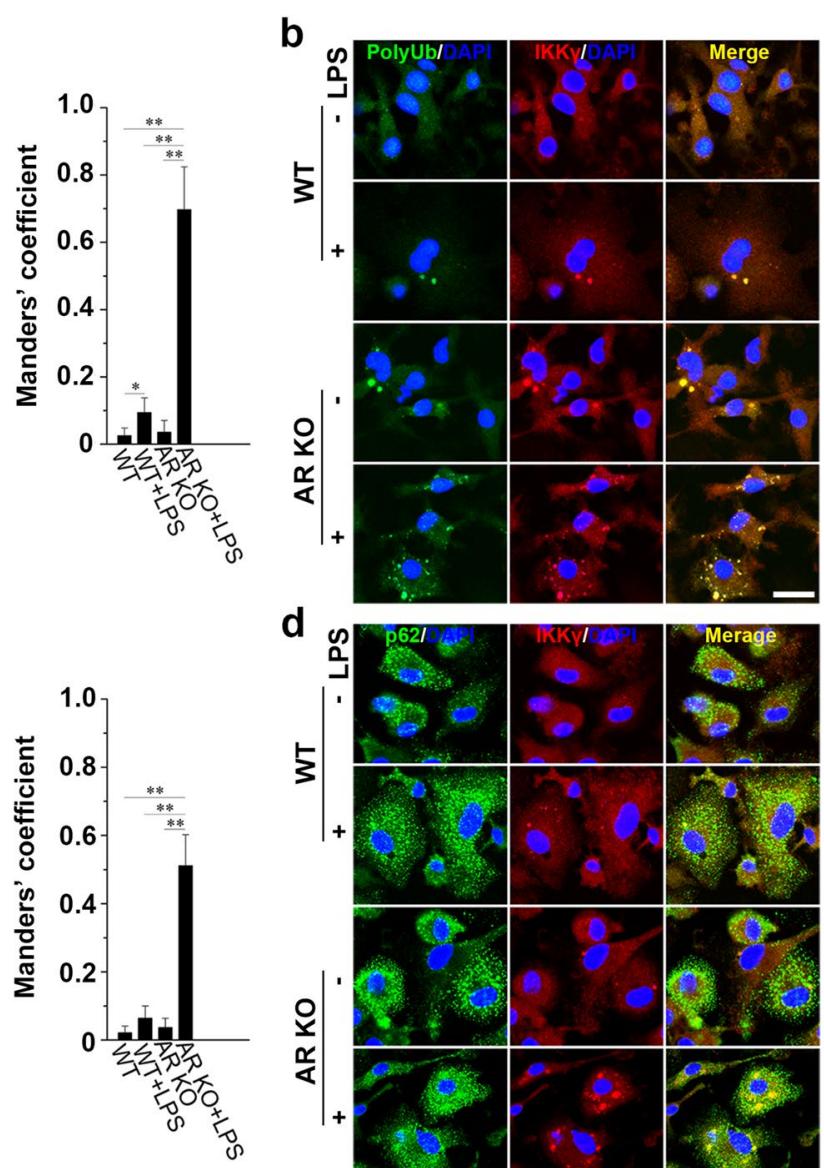

d

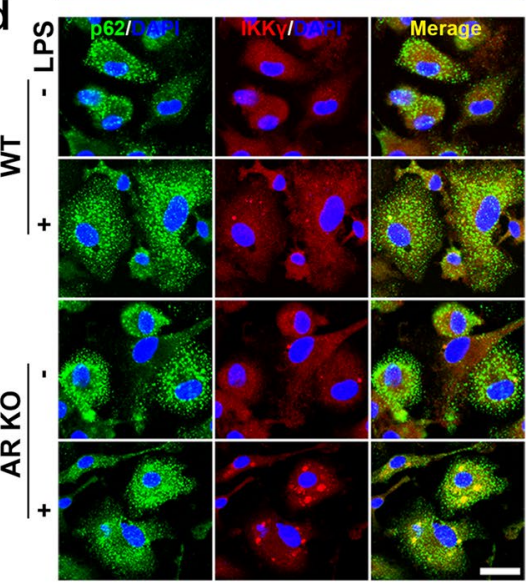

Fig. 6 Connecting the IKK complex and autophagy pathway. Confocal microscopy images of BMMs from WT or AR KO mice treated with or without $500 \mathrm{ng} / \mathrm{ml} \mathrm{LPS}$ for $1 \mathrm{~h}$, and co-stained for a polyUb and IKKß, b polyUb and IKKY, c p62 and IKKß, and $\mathbf{d}$ p62 and IKKY. Right graphs represent the quantification of co-localization of signals using Manders' overlap coefficient. Scale bars $=20 \mu \mathrm{m}$. Data are expressed as the mean \pm SD from three independent experiments, ${ }^{*} P<0.05,{ }^{* *} P<0.01$

polyubiquitination has also been associated with the formation and autophagic degradation of protein inclusions [43]. Interestingly, the preferential binding of p62 to K63linked chains constitute a further level of regulation during selective autophagy [44].

Confocal microscopy of BMMs immunostained for PolyUb (linkage-specific K63) and IKK $\beta$ showed that in the absence of LPS stimulation, ubiquitin resided mainly in the cytosol where it appeared as small PolyUb ${ }^{+}$dots, whereas IKK $\beta$ showed an occasional granular pattern (Fig. 6a). Treatment of BMMs with LPS for $1 \mathrm{~h}$ resulted in the formation of large IKK $\beta$ aggregates co-localized with PolyUb (Fig. 6a). Confocal microscopy results further suggested that in stimulated WT cells, only a small fraction of IKK $\beta$ was co-localized with PolyUb ${ }^{+}$; in contrast, the overlaps between IKK $\beta$ and PolyUb ${ }^{+}$dots in LPStreated AR KO cells were markedly increased (Fig. 6a). Similarly, we found that LPS stimulation induced the recruitment of $I K K \gamma$ to PolyUb ${ }^{+}$structures, and that the co-localization of IKK $\gamma$ and PolyUb was markedly increased in AR KO cells (Fig. 6b).

Next, we treated primary macrophages with or without LPS and immunostained the cells for p62 and IKK $\beta$. We noted that only a small fraction of $\mathrm{p} 62$ was associated with IKK $\beta$ in non-stimulated and LPS-treated WT cells, but this association was greatly increased in LPSstimulated AR-deficient BMMs (Fig. 6c). Consistently, LPS-induced interactions between IKK $\gamma$ and p62 were promoted by the knockout of AR in BMMs (Fig. 6d). These data indicated that IKK $\beta$ and IKK $\gamma$ aggregates were polyubiquitinated and that they could be targeted by $\mathrm{p} 62$ to the autophagy pathway in LPS-induced AR-deficient macrophages.

\section{Discussion}

Our results showed that AR deficiency led to the suppression of the M1 phenotype in macrophages, limiting its pro-inflammatory activity through the degradation of a key 
component of the IKK complex. Knockout of AR triggered autophagosome formation, and the exposure of AR KO macrophages to LPS markedly enhanced autophagosome biogenesis and maturation. Autophagy was able to capture and degrade IKK $\beta$ and IKK $\gamma$ through polyubiquitination, leading to the recruitment of p62 and LC3. Autophagosome formation in the AR knockout background was functionally important; pharmacological blockade of autophagy pathway increased the protein levels of IKK $\beta$ and IKK $\gamma$ and enhanced the production of iNOS. These data suggested a close relationship among AR, the M1 response, and autophagy.

There are two main NF-kB pathways in the cell. The noncanonical NF- $\mathrm{KB}$ signaling pathway depends on IKK $\alpha$ and plays a critical role in the development of lymphoid organs responsible for the generation of $\mathrm{B}$ and $\mathrm{T}$ lymphocytes [45]. Neither IKK $\beta$ nor IKK $\gamma$ deficiency affects this pathway [45]. However, the canonical pathway, induced by most physiological NF- $\mathrm{kB}$ stimuli, depends on IKK $\beta$ and IKK $\gamma$. The activation of this pathway mainly leads to the phosphorylation of IкB $\alpha$ and the nuclear translocation of mostly p65-containing heterodimers [28, 46]. However, IKK $\gamma$ deficiency appears to have a greater impact on the classical NF- $\mathrm{kB}$ signaling pathway than IKK $\beta$ deficiency [45]. It has been reported that cells lacking IKK $\gamma$ have no detectable NF-kB response to almost any pro-inflammatory or immune regulatory stimuli [47]. Our results showed that the exposure of AR KO macrophages to LPS impaired both IKK $\beta$ and IKK $\gamma$. This may be attributed to the suppression of the TLR4/NF- $\mathrm{kB}$ signaling pathway, iNOS induction, and M1 polarization in AR-deficient BMMs.

We investigated the mechanism through which certain signals downregulate the IKK $\beta$ and IKK $\gamma$ proteins without affecting their mRNA levels in AR KO macrophages after LPS treatment. We speculated that IKK $\beta$ and IKKY protein levels were decreased at the post-transcriptional level in AR KO macrophages upon LPS stimulation. Previous studies showed that 4-hydroxynonenal (4-HNE) is an endogenous substrate for AR, with a higher affinity than glucose $[48,49]$. Exogenous 4-HNE triggers autophagy, and deletion of AR increases 4-HNE accumulation and autophagy [17, 50]. We also found that the exposure of AR KO macrophages to LPS markedly increased the level of 4-HNE (Additional file 1: Fig. S1) and enhanced autophagosome formation. Furthermore, we investigated the relationship between the impairment of the IKK complex and autophagosome formation. We observed that a massive co-localization of IKK $\beta$, IKK $\gamma$, $\mathrm{LC}^{+}$autophagic vacuoles, and LAMP1 ${ }^{+}$lysosomes happens in BMMs from AR KO mice after LPS stimulation. Furthermore, our finding that AR deficiency led to the polyubiquitination of IKK $\beta$ and IKK $\gamma$ provides a possible answer. K63-ubiquitinated IKK $\beta$ and IKK $\gamma$ can recruit p62, which can deliver them to the autophagy pathway through its LC3-binding domain. Conversely, pharmacological suppression of autophagy partially restored the protein levels of IKK $\beta$ and IKK $\gamma$ as well as the induction of iNOS. Thus, manipulation of AR expression or activity may have a therapeutic potential by regulating macrophage polarization in inflammatory diseases.

\section{Supplementary Information}

The online version contains supplementary material available at https://doi. org/10.1186/s13578-021-00576-7.

Additional file 1: Figure S1. LPS stimulation greatly alters the level of 4-HHE in AR KO macrophages.

\section{Acknowledgements}

We thank Professor Sookja Kim Chung for providing us the AR KO mice. We would like to thank Editage (www.editage.cn) for English language editing.

\section{Authors' contributions}

PC and JW conceived and designed the experiments. PC, JWX and ZYL performed the experiments. PC and JW wrote and edited the manuscript. All authors read and approved the final manuscript.

\section{Funding}

This study was supported by Key Research and Development Project of Hainan Province [ZDYF2019168].

Availability of data and materials

All data generated or analyzed during this study are included in this published article.

\section{Declarations}

Ethics approval and consent participate

The animal studies were approved by the Animal Care and Use Committee of Fourth Military Medical University and were conducted in accordance with the National Institutes of Health guidelines for the care and use of laboratory animals.

\section{Consent for publication}

Not applicable.

\section{Competing interests}

The authors declare that they have no competing interests.

Received: 11 December 2020 Accepted: 19 March 2021

Published online: 26 March 2021

\section{References}

1. Sica A, Mantovani A. Macrophage plasticity and polarization: in vivo veritas. J Clin Invest. 2012;122(3):787-95.

2. Murray PJ, Wynn TA. Protective and pathogenic functions of macrophage subsets. Nat Rev Immunol. 2011;11(11):723-37.

3. Funes SC, Rios M, Escobar-Vera J, Kalergis AM. Implications of macrophage polarization in autoimmunity. Immunology. 2018;154(2):186-95

4. Ramana KV, Srivastava SK. Aldose reductase: a novel therapeutic target for inflammatory pathologies. Int J Biochem Cell Biol. 2010;42(1):17-20.

5. Ravindranath TM, Mong PY, Ananthakrishnan R, Li Q, Quadri N, Schmidt AM, Ramasamy R, et al. Novel role for aldose reductase in mediating acute inflammatory responses in the lung. J Immunol. 2009:183(12):8128-37. 
6. Shoeb M, Yadav UC, Srivastava SK, Ramana KV. Inhibition of aldose reductase prevents endotoxin-induced inflammation by regulating the arachidonic acid pathway in murine macrophages. Free Radic Biol Med. 2011;51(9):1686-96.

7. Yadav UC, Ramana KV, Aguilera-Aguirre L, Boldogh I, Boulares HA, Srivastava SK. Inhibition of aldose reductase prevents experimental allergic airway inflammation in mice. PLoS ONE. 2009;4(8):e6535.

8. Ramana KV, Srivastava SK. Mediation of aldose reductase in lipopolysaccharide-induced inflammatory signals in mouse peritoneal macrophages. Cytokine. 2006;36(3-4):115-22.

9. Ballekova J, Soltesova-Prnova M, Majekova M, Stefek M. Does inhibition of aldose reductase contribute to the anti-inflammatory action of setipiprant? Physiol Res. 2017;66(4):687-93.

10. Yadav UC, Shoeb M, Srivastava SK, Ramana KV. Aldose reductase deficiency protects from autoimmune- and endotoxin-induced uveitis in mice. Invest Ophthalmol Vis Sci. 2011;52(11):8076-85.

11. Erbel C, Rupp G, Domschke G, Linden F, Akhavanpoor M, Doesch AO, Katus HA, et al. Differential regulation of aldose reductase expression during macrophage polarization depends on hyperglycemia. Innate Immun. 2016;22(3):230-7.

12. Luo D, Guo Y, Cheng Y, Zhao J, Wang Y, Rong J. Natural product celastrol suppressed macrophage $\mathrm{M} 1$ polarization against inflammation in diet-induced obese mice via regulating Nrf2/HO-1, MAP kinase and NFkappaB pathways. Aging. 2017;9(10):2069-82.

13. Chiang CF, Chao TT, Su YF, Hsu CC, Chien CY, Chiu KC, Shiah SG, et al. Metformin-treated cancer cells modulate macrophage polarization through AMPK-NF-kappaB signaling. Oncotarget. 2017;8(13):20706-18.

14. Tian L, Li W, Yang L, Chang N, Fan X, Ji X, Xie J, et al. Cannabinoid receptor 1 participates in liver inflammation by promoting $M 1$ macrophage polarization via RhoA/NF-kappaB p65 and ERK1/2 pathways, respectively, in mouse liver fibrogenesis. Front Immunol. 2017;8:1214.

15. He Y, Ma X, Li D, Hao J. Thiamet G mediates neuroprotection in experimental stroke by modulating microglia/macrophage polarization and inhibiting NF-kappaB p65 signaling. J Cereb Blood Flow Metab. 2017;37(8):2938-51.

16. Zhang Q, Bian G, Chen P, Liu L, Yu C, Liu F, Xue Q, et al. Aldose reductase regulates microglia/macrophages polarization through the CAMP response element-binding protein after spinal cord injury in mice. Mol Neurobiol. 2016;53(1):662-76.

17. Baba SP, Zhang D, Singh M, Dassanayaka S, Xie Z, Jagatheesan G, Zhao J, et al. Deficiency of aldose reductase exacerbates early pressure overloadinduced cardiac dysfunction and autophagy in mice. J Mol Cell Cardiol. 2018;118:183-92.

18. Zhang D, Bhatnagar A, Baba SP. Inhibition of aldose reductase activity stimulates starvation induced autophagy and clears aldehyde protein adducts. Chem Biol Interact. 2019;306:104-9.

19. Glick D, Barth S, Macleod KF. Autophagy: cellular and molecular mechanisms. J Pathol. 2010;221(1):3-12.

20. Shi CS, Shenderov K, Huang NN, Kabat J, Abu-Asab M, Fitzgerald KA, Sher $A$, et al. Activation of autophagy by inflammatory signals limits IL-1 beta production by targeting ubiquitinated inflammasomes for destruction. Nat Immunol. 2012;13(3):255-63.

21. Xu Y, Jagannath C, Liu XD, Sharafkhaneh A, Kolodziejska KE, Eissa NT. Tolllike receptor 4 is a sensor for autophagy associated with innate immunity. Immunity. 2007;27(1):135-44.

22. Tateyama $H$, Murase $Y$, Higuchi $H$, Inasaka $Y$, Kaneoka $H$, lijima S, Nishijima $\mathrm{Kl}$. Siglec-F is induced by granulocyte-macrophage colony-stimulating factor and enhances interleukin-4-induced expression of arginase-1 in mouse macrophages. Immunology. 2019;158(4):340-52.

23. Bolte $\mathrm{S}$, Cordelieres FP. A guided tour into subcellular colocalization analysis in light microscopy. J Microsc. 2006;224(Pt 3):213-32.

24. Cheng P, Kuang F, Ju G. Aescin reduces oxidative stress and provides neuroprotection in experimental traumatic spinal cord injury. Free Radic Biol Med. 2016;99:405-17.

25. McWhorter FY, Davis CT, Liu WF. Physical and mechanical regulation of macrophage phenotype and function. Cell Mol Life Sci. 2015;72(7):1303-16.

26. Musial A, Eissa NT. Inducible nitric-oxide synthase is regulated by the proteasome degradation pathway. J Biol Chem. 2001;276(26):24268-73.
27. Gray CM, Remouchamps C, McCorkell KA, Solt LA, Dejardin E, Orange JS, May MJ. Noncanonical NF-kappaB signaling is limited by classical NFkappaB activity. Sci Signal. 2014;7(311):ra13.

28. Bonizzi G, Karin M. The two NF-kappaB activation pathways and their role in innate and adaptive immunity. Trends Immunol. 2004;25(6):280-8.

29. Adli M, Merkhofer E, Cogswell P, Baldwin AS. IKKalpha and IKKbeta each function to regulate NF-kappaB activation in the TNF-induced/canonical pathway. PLoS ONE. 2010;5(2):e9428.

30. Rothwarf DM, Zandi E, Natoli G, Karin M. IKK-gamma is an essential regulatory subunit of the IkappaB kinase complex. Nature. 1998;395(6699):297-300.

31. Shi CS, Kehrl JH. MyD88 and Trif target Beclin 1 to trigger autophagy in macrophages. J Biol Chem. 2008;283(48):33175-82.

32. Tanida I. Autophagosome formation and molecular mechanism of autophagy. Antioxid Redox Signal. 2011;14(11):2201-14.

33. Tanida I, Minematsu-Ikeguchi N, Ueno T, Kominami E. Lysosomal turnover, but not a cellular level, of endogenous LC3 is a marker for autophagy. Autophagy. 2005;1(2):84-91.

34. Tanida I, Ueno T, Kominami E. LC3 conjugation system in mammalian autophagy. Int J Biochem Cell Biol. 2004;36(12):2503-18.

35. Tanida I, Waguri S. Measurement of autophagy in cells and tissues. Methods Mol Biol. 2010;648:193-214.

36. Zhao YG, Zhang $\mathrm{H}$. Autophagosome maturation: an epic journey from the ER to lysosomes. J Cell Biol. 2019;218(3):757-70.

37. Pasquier B. Autophagy inhibitors. Cell Mol Life Sci. 2016;73(5):985-1001.

38. Calvo-Garrido J, Carilla-Latorre S, Mesquita A, Escalante R. A proteolytic cleavage assay to monitor autophagy in Dictyostelium discoideum. Autophagy. 2011;7(9):1063-8.

39. Kihara A, Noda T, Ishihara N, Ohsumi Y. Two distinct Vps34 phosphatidylinositol 3-kinase complexes function in autophagy and carboxypeptidase $Y$ sorting in Saccharomyces cerevisiae. J Cell Biol. 2001;152(3):519-30.

40. Lamark T, Svenning $S$, Johansen T. Regulation of selective autophagy: the p62/SQSTM1 paradigm. Essays Biochem. 2017;61(6):609-24.

41. Katsuragi $Y$, Ichimura Y, Komatsu M. p62/SQSTM1 functions as a signaling hub and an autophagy adaptor. FEBS J. 2015;282(24):4672-8.

42. Fujita K, Srinivasula SM. TLR4-mediated autophagy in macrophages is a p62-dependent type of selective autophagy of aggresome-like induced structures (ALIS). Autophagy. 2011;7(5):552-4.

43. Tan JM, Wong ES, Kirkpatrick DS, Pletnikova O, Ko HS, Tay SP, Ho MW, et al. Lysine 63-linked ubiquitination promotes the formation and autophagic clearance of protein inclusions associated with neurodegenerative diseases. Hum Mol Genet. 2008;17(3):431-9.

44. Zaffagnini G, Martens S. Mechanisms of Selective Autophagy. J Mol Biol. 2016;428(9 Pt A):1714-24.

45. Liu F, Xia Y, Parker AS, Verma IM. IKK biology. Immunol Rev. 2012;246(1):239-53.

46. Oeckinghaus A, Hayden MS, Ghosh S. Crosstalk in NF-kappaB signaling pathways. Nat Immunol. 2011;12(8):695-708.

47. Yamaoka S, Courtois G, Bessia C, Whiteside ST, Weil R, Agou F, Kirk HE, et al. Complementation cloning of NEMO, a component of the IkappaB kinase complex essential for NF-kappaB activation. Cell. 1998;93(7):1231-40.

48. Srivastava S, Dixit BL, Cai J, Sharma S, Hurst HE, Bhatnagar A, Srivastava SK. Metabolism of lipid peroxidation product, 4-hydroxynonenal (HNE) in rat erythrocytes: role of aldose reductase. Free Radic Biol Med. 2000;29(7):642-51.

49. Vander Jagt DL, Kolb NS, Vander Jagt TJ, Chino J, Martinez FJ, Hunsaker LA, Royer RE. Substrate specificity of human aldose reductase: identification of 4-hydroxynonenal as an endogenous substrate. Biochim Biophys Acta. 1995;1249(2):117-26.

50. Hill BG, Haberzettl P, Ahmed Y, Srivastava S, Bhatnagar A. Unsaturated lipid peroxidation-derived aldehydes activate autophagy in vascular smooth-muscle cells. Biochem J. 2008;410(3):525-34.

\section{Publisher's Note}

Springer Nature remains neutral with regard to jurisdictional claims in published maps and institutional affiliations. 